\title{
Difference between Irregular Chaotic Patterns of Second-order Double-loop $\Sigma \Delta$ Modulators and Second-order Interpolative Bandpass $\Sigma \Delta$ Modulators
}

\author{
Charlotte Yuk-Fan Ho \\ Telephone: 44 (0)20 78827986 Fax: 44 (0)20 78827997 Email: charlotte.ho@elec.qmul.ac.uk \\ Department of Electronic Engineering, Queen Mary, University of London, Mile End Road, London, \\ E1 4NS, United Kingdom.
}

Bingo Wing-Kuen Ling

Telephone: 44 (0)20 78482294 Fax: 44 (0)20 78482932 Email: wing-kuen.ling@kcl.ac.uk Department of Electronic Engineering, Division of Engineering, King's College London, Strand, London, WC2R 2LS, United Kingdom.

Joshua D. Reiss

Telephone: 44 (0)20 78827982 Fax: 44 (0)20 78827997 Email: josh.reiss@elec.qmul.ac.uk Department of Electronic Engineering, Queen Mary, University of London, Mile End Road, London, E1 4NS, United Kingdom.

\begin{abstract}
In this paper, we find that, by computing the difference between two consecutive state vectors of second-order double-loop sigma-delta modulators (SDMs) and plotting one component of the subtracted vectors against the other component, irregular chaotic patterns will become two vertical lines. By multiplying a matrix on the subtracted vectors, it can be further transformed to two fixed points. However, second-order interpolative bandpass SDMs still exhibit chaotic behaviors after applying the same transformations. Moreover, it is found that the Lyapunov exponent of state vectors of second-order double-loop SDMs is higher than that of second-order interpolative bandpass SDMs, whereas the Lyapunov exponent of transformed vectors becomes negative infinity for second-order double-loop SDMs and increases for second-order interpolative bandpass SDMs. Hence, by examining the occurrence of chaotic behaviors of the transformed vectors of these two SDMs, these two SDMs can be distinguished from their state vectors and their transformed
\end{abstract}


vectors without solving the state equations and knowing the information of input signals.

\section{INTRODUCTION}

It was well known that there are many important applications of SDMs in analog-to-digital conversions because only simple, robust and inexpensive components can achieve the objectives [1]-[3]. However, since SDMs consist of discontinuous nonlinear elements, which are quantizers, behaviors of SDMs could be very complex even though SDMs are as simple as second-order systems [4]-[5]. One of the most common complex behaviors is the exhibition of irregular chaotic patterns on the phase portraits [4].

However, since both second-order double-loop SDMs and second-order interpolative bandpass SDMs would exhibit irregular chaotic patterns on the phase portraits, it is not easy to distinguish these two types of SDMs from their state vectors without solving the state equations and knowing the information of input signals. This problem is important because SDMs are sometimes disturbed by a mechanical shaking or an electric shocking. In these situations, filter parameters in SDMs may be corrupted. In order to reconstruct signals, it is required to estimate the corresponding filter parameters. Although there are infinite numbers of choices of filter parameters, the most common one is either from second-order double-loop SDMs or second-order interpolative bandpass SDMs. By distinguishing these two types of SDMs, signals can be reconstructed.

Although there are some existing methods for solving nonlinear system identification problems, such as using the fuzzy [7] and neural network [8] approaches, these approaches are too complicated for the identification of SDMs. This is because input signals are usually oversampled and the number of discrete samples is very large. Hence, the computational complexity is too high for these methods which make them practically impossible. On the other hand, properties of SDMs are exploited in this paper. The proposed method is less computational complex compared to the fuzzy [7] and neural network [8] approaches. Hence, it can be applied to distinguish these two types of SDMs from their state vectors.

The outline of this paper is as follows. In Section II, we show that, after computing the difference between two consecutive state vectors of second-order double-loop SDMs, there are only two vertical lines by plotting the component of the 
vectors against the other component, whereas, this is not the case for second-order interpolative bandpass SDMs. In Section III, we explain why this method can be used to distinguish these two types of SDMs from their state vectors without solving the state equations and knowing the information of input signals. Finally, the conclusion and summary of our works are given in Section IV.

\section{RESULTS}

For a lowpass SDM or a bandpass SDM with the natural frequency of the loop filter closed to zero, input signals are usually slow time varying and can be approximated by a step signal because the oversampling ratio is usually very high. Hence, a step input can be considered in this paper. For the second-order double-loop SDM shown in [4], the dynamical behavior of the SDM can be represented by the following state space equation:

$$
\mathbf{x}(k+1)=\mathbf{A x}(k)+\mathbf{B s}(k)+u\left[\begin{array}{ll}
1 & 1
\end{array}\right]^{T},
$$

for $\quad k \geq 0$, where $\quad \mathbf{x}(k) \equiv\left[x_{1}(k) \quad x_{2}(k)\right]^{T} \quad$ is the state vector, $\mathbf{s}(k) \equiv\left[s_{1}(k) \quad s_{2}(k)\right]^{T} \equiv Q(\mathbf{x}(k))$ is the quantized state vector, $u$ is the input step size,

$$
\mathbf{A} \equiv\left[\begin{array}{ll}
1 & 0 \\
1 & 1
\end{array}\right], \mathbf{B} \equiv\left[\begin{array}{ll}
0 & -1 \\
0 & -2
\end{array}\right] \text { and } Q(y) \equiv\left\{\begin{array}{cc}
1 & y \geq 0 \\
-1 & \text { otherwise }
\end{array} .\right.
$$

Define

$$
\mathbf{e}(k) \equiv \mathbf{x}(k+1)-\mathbf{x}(k),
$$

then it can be shown easily that

$$
\mathbf{e}(k) \equiv\left[e_{1}(k) \quad e_{2}(k)\right]^{T}=\left[u-s_{2}(k) \quad x_{1}(k)+u-2 s_{2}(k)\right]^{T} .
$$

Since $u$ is a constant and $s_{i}(k) \in\{1,-1\}$ for $i=1,2$,

$$
e_{1}(k) \in\{u-1, u+1\}
$$

and

$$
\min _{\forall k}\left(x_{1}(k)\right)+u-2 \leq e_{2}(k) \leq \max _{\forall k}\left(x_{1}(k)\right)+u+2 .
$$

If $x_{1}(k)$ is bounded, then $e_{2}(k)$ is also bounded, and there are two vertical lines shown on the plot of $e_{2}(k)$ against $e_{1}(k)$.

It is worth noting that $\mathbf{e}(k)$ is obtained by highpass filtering of $\mathbf{x}(k)$. Hence, the mapping from $X \equiv\{\mathbf{x}(k)\}$ to $E \equiv\{\mathbf{e}(k)\}$ is not a static projection of $\mathbf{x}(k)$. Furthermore, define 


$$
\widetilde{\mathbf{e}}(k) \equiv\left[\tilde{e}_{1}(k) \quad \tilde{e}_{2}(k)\right]^{T} \equiv(\mathbf{A}-\mathbf{I}) \mathbf{e}(k),
$$

where $\mathbf{I}$ is a $2 \times 2$ identity matrix, then

$$
\tilde{\mathbf{e}}(k)=\left[\begin{array}{ll}
0 & u-s_{2} \\
(k)
\end{array}\right]^{T} .
$$

In this case, $\tilde{\mathbf{e}}(k)$ is obtained from a static projection of $\mathbf{e}(k)$. Since there are only two possible values of $\widetilde{\mathbf{e}}(k)$, which are $\left[\begin{array}{ll}0 & u-1\end{array}\right]^{T}$ and $\left[\begin{array}{ll}0 & u+1\end{array}\right]^{T}$, there are only two points on the plot of $\tilde{e}_{2}(k)$ against $\tilde{e}_{1}(k)$.

Figure 1a shows the phase portrait of a second-order double-loop SDM with $u=0.161027$ and $\mathbf{x}(0)=\left[\begin{array}{ll}0.077 & 0.08\end{array}\right]^{T} \quad$ [4]. Figure $1 \mathrm{~b}$ shows the plot of $e_{2}(k)$ against $e_{1}(k)$. Figure $1 \mathrm{c}$ shows the plot of $\tilde{e}_{2}(k)$ against $\tilde{e}_{1}(k)$. It can be seen from Figure 1a that an irregular chaotic pattern is exhibited on the phase portrait, while there are two vertical lines on the plot of $e_{2}(k)$ against $e_{1}(k)$, and two points on the plot of $\tilde{e}_{2}(k)$ against $\tilde{e}_{1}(k)$.
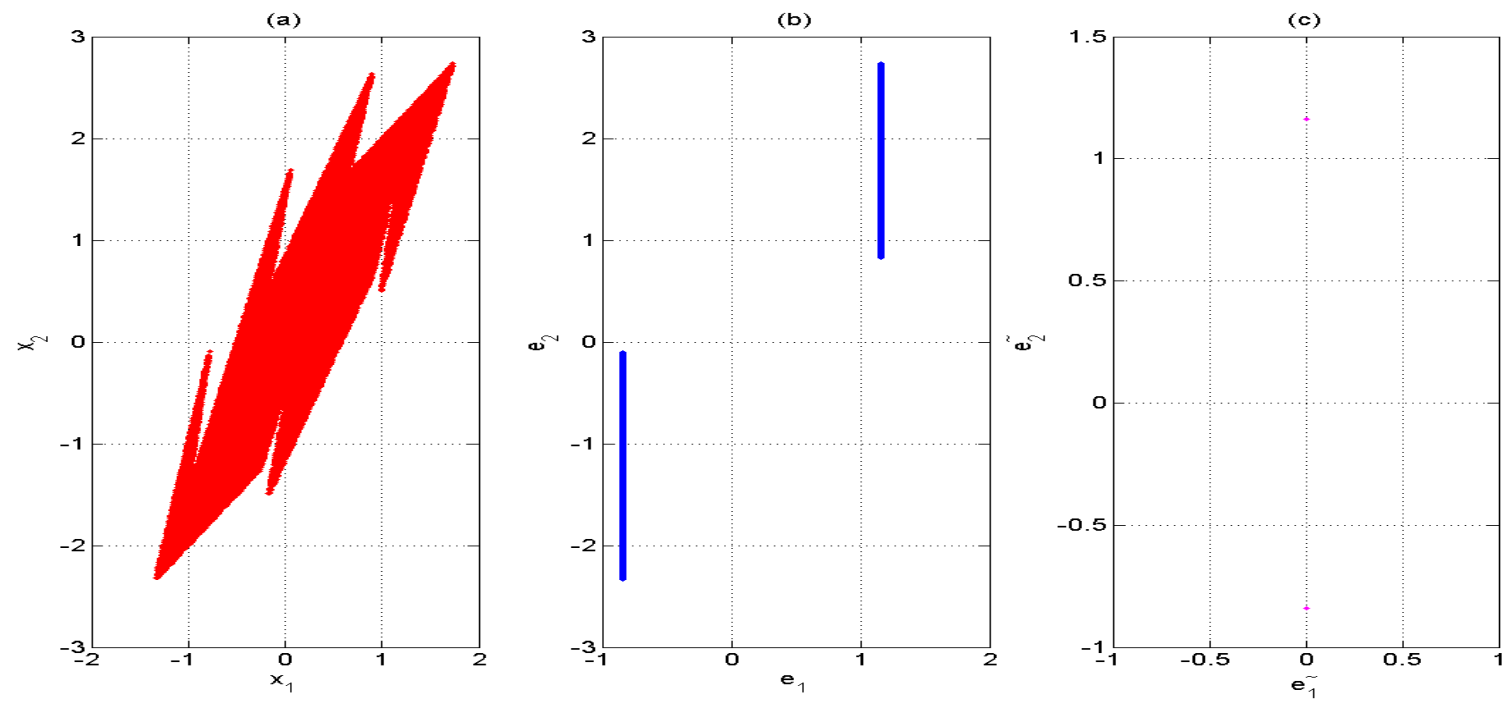

Figure 1: (a) Phase portrait of a second-order double-loop SDM. (b) Plot of $e_{2}(k)$

$$
\text { against } e_{1}(k) \text {. (c) Plot of } \tilde{e}_{2}(k) \text { against } \tilde{e}_{1}(k) \text {. }
$$

Now, we apply the same transformation to second-order interpolative bandpass SDMs [5]. Second-order interpolative bandpass SDMs can be represented by the following state space equation:

$$
\mathbf{x}(k+1)=\mathbf{A}^{\prime} \mathbf{x}(k)+\mathbf{B}^{\prime}\left(u\left[\begin{array}{ll}
1 & 1
\end{array}\right]^{T}-\mathbf{s}(k)\right),
$$

for $k \geq 0$, where

$$
\mathbf{A}^{\prime} \equiv\left[\begin{array}{cc}
0 & 1 \\
-1 & 2 \cos \theta
\end{array}\right] \text { and } \mathbf{B}^{\prime} \equiv\left[\begin{array}{cc}
0 & 0 \\
d & -2 d \cos \theta
\end{array}\right]
$$


Figure 2a shows the phase portrait when $d=-1, \theta=0.05, u=-0.3$ and $\mathbf{x}(0)=\left[\begin{array}{ll}0.1 & -0.5\end{array}\right]^{T}$. Figure 2b shows the plot of $e_{2}(k)$ against $e_{1}(k)$, and Figure 2c shows the plot of $\tilde{e}_{2}(k)$ against $\tilde{e}_{1}(k)$. It can be seen from Figure 2a that an irregular chaotic pattern is exhibited on the phase portrait, while it seems to have four straight lines on the plot of $e_{2}(k)$ against $e_{1}(k)$ as shown in Figure $2 \mathrm{~b}$ and four points on the plot of $\tilde{e}_{2}(k)$ against $\tilde{e}_{1}(k)$ as shown in Figure 2c. However, if we zoom-in into Figure $2 \mathrm{~b}$ and Figure 2c, as shown in Figure $2 \mathrm{~d}$ and 2e, we find that they are not four straight lines and four points. In fact, the trajectories are confined in very narrow regions within the neighborhood of four straight lines and four points respectively. The width of the regions depends on $\theta$. The Lyapunov exponents of $\mathbf{x}(k)$ and $\tilde{\mathbf{e}}(k)$ for the second-order interpolative bandpass SDM are, respectively, 0.0619 and 0.0628, where the Lyapunov exponent is computed based on 50,000 samples of data and 30 total number of replacement steps [6]. It is worth noting that the Lyapunov exponent of $\tilde{\mathbf{e}}(k)$ is higher than that of $\mathbf{x}(k)$. If the Lyapunov exponent is employed for the measure of chaotic behaviors, since both Lyapunov exponents are positive, then $\mathbf{x}(k)$ and $\tilde{\mathbf{e}}(k)$ can be regarded as exhibiting chaotic behaviors and $\widetilde{\mathbf{e}}(k)$ is more chaotic than $\mathbf{x}(k)$ (in the sense of the Lyapunov exponent). Compared to that of $\mathbf{x}(k)$ and $\tilde{\mathbf{e}}(k)$ for the second-order double-loop SDM, the corresponding values are 0.2341 and $-\infty$, respectively. Since the Lyapunov exponent of $\mathbf{x}(k)$ for the second-order doubleloop SDM is higher than that of the second-order interpolative bandpass SDM, while the Lyapunov exponent of $\widetilde{\mathbf{e}}(k)$ is negative and much smaller than that of the secondorder interpolative bandpass SDM, we can conclude that the state vectors of the second-order double-loop SDM is more chaotic than that of the second-order interpolative bandpass SDM, but it is no longer chaotic after the transformation, while the transformation causes more chaotic for the second-order interpolative bandpass SDM.

The importance of the above findings is that we can distinguish these two types of SDMs from their state vectors without solving the state equations and the information of input signals. 


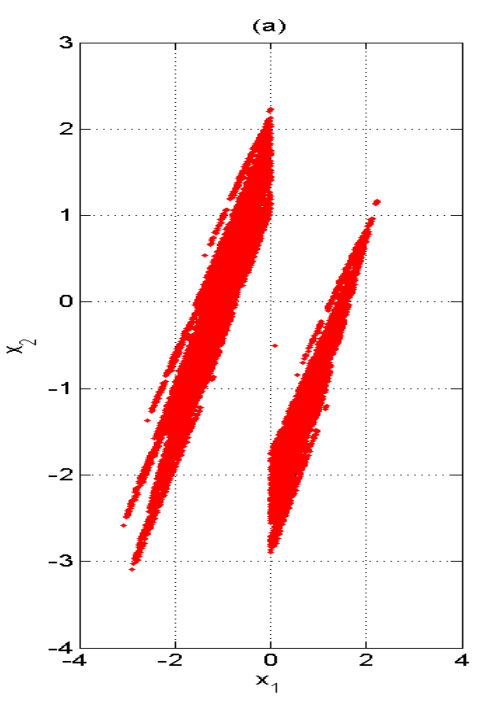

(d)

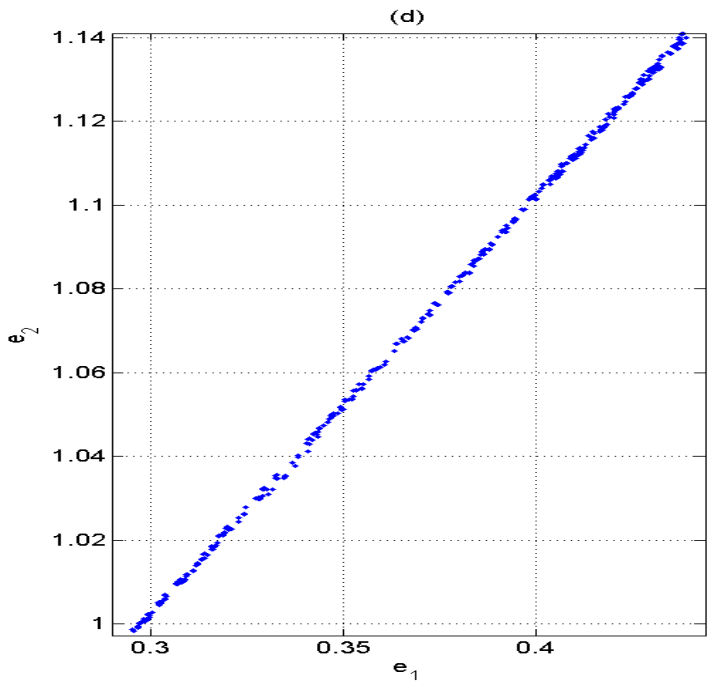

(b)
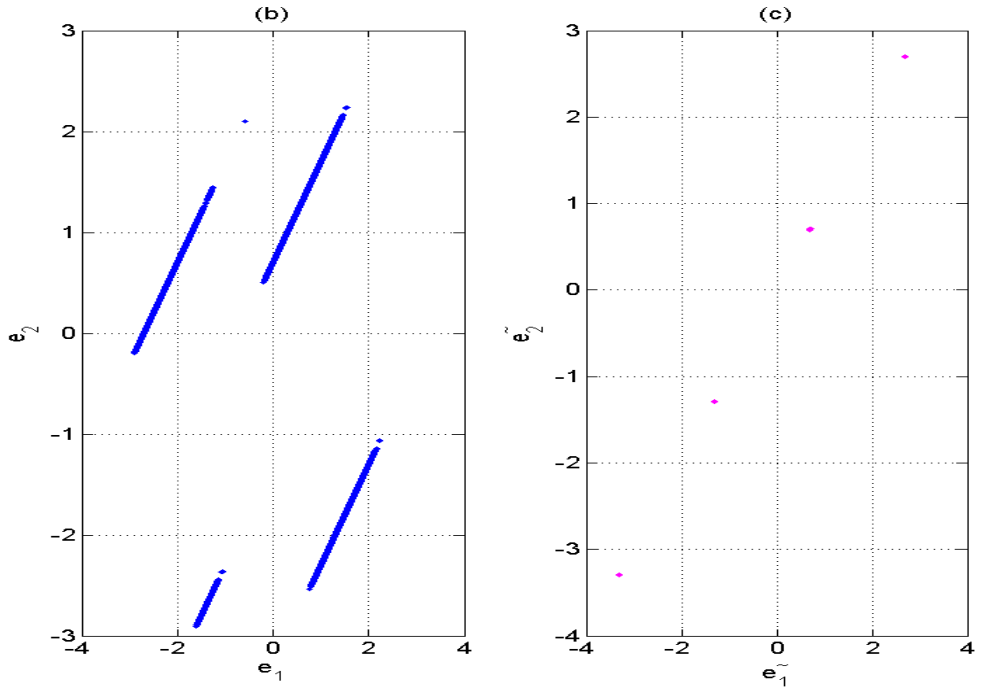

(e)

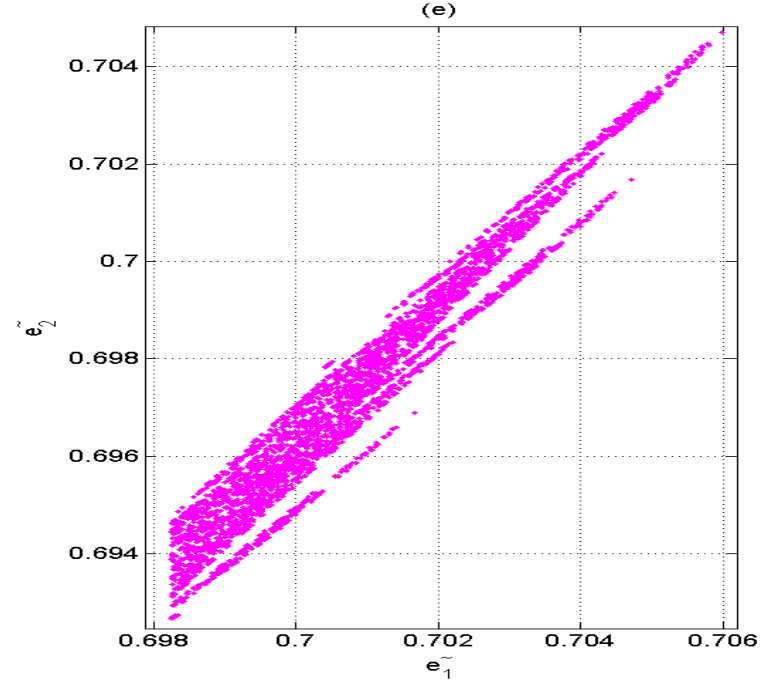

Figure 2: (a) Phase portrait of a second-order interpolative bandpass SDM. (b) Plot of $e_{2}(k)$ against $e_{1}(k)$. (c) Plot of $\tilde{e}_{2}(k)$ against $\tilde{e}_{1}(k)$. (d) Zoom-in of (b). (e) Zoom-in of (e).

\section{EXPLANATIONS}

For second-order double-loop SDMs,

$$
\mathbf{e}(k)=(\mathbf{A}-\mathbf{I}) \mathbf{x}(k)+\mathbf{B s}(k)+u\left[\begin{array}{ll}
1 & 1
\end{array}\right]^{T},
$$

for $k \geq 0$, and

$$
\tilde{\mathbf{e}}(k)=(\mathbf{A}-\mathbf{I})^{2} \mathbf{x}(k)+(\mathbf{A}-\mathbf{I})\left(\mathbf{B s}(k)+u\left[\begin{array}{ll}
1 & 1
\end{array}\right]^{T}\right)
$$

for $k \geq 0$. Since

$$
\mathbf{A}-\mathbf{I}=\left[\begin{array}{ll}
0 & 0 \\
1 & 0
\end{array}\right]
$$

and 


$$
(\mathbf{A}-\mathbf{I})^{2}=\mathbf{0},
$$

the matrix $\mathbf{A}-\mathbf{I}$ is not a full rank matrix. Hence, the dimension of the signal is reduced after the projection. As a result, exactly two vertical lines and two points are exhibited on the plots of $e_{2}(k)$ against $e_{1}(k)$, and $\tilde{e}_{2}(k)$ against $\tilde{e}_{1}(k)$, respectively. For second-order interpolative bandpass SDMs, since

$$
\mathbf{A}^{\prime}-\mathbf{I}=\left[\begin{array}{cc}
-1 & 1 \\
-1 & 2 \cos \theta-1
\end{array}\right]
$$

and

$$
\left(\mathbf{A}^{\prime}-\mathbf{I}\right)^{2}=\left[\begin{array}{cc}
0 & 2(\cos \theta-1) \\
2(1-\cos \theta) & (2 \cos \theta-1)^{2}-1
\end{array}\right],
$$

the matrix $\mathbf{A}^{\prime}-\mathbf{I}$ is a full rank matrix, the dimension of the signals is preserved after the projection. Hence, they are not four straight lines and four points on the plots of $e_{2}(k)$ against $e_{1}(k)$, and $\tilde{e}_{2}(k)$ against $\tilde{e}_{1}(k)$, respectively. However, if $\theta$ is small enough, that is $\theta \rightarrow 0$, then

$$
\mathbf{A}^{\prime}-\mathbf{I} \approx\left[\begin{array}{ll}
-1 & 1 \\
-1 & 1
\end{array}\right]
$$

and

$$
\left(\mathbf{A}^{\prime}-\mathbf{I}\right)^{2} \approx \mathbf{0} .
$$

In this case, it appears to be four straight lines and four points on the plot of $e_{2}(k)$ against $e_{1}(k)$ and $\widetilde{e}_{2}(k)$ against $\widetilde{e}_{1}(k)$, respectively. This accounts for the phenomena.

\section{CONCLUSION}

In this paper, we have explored the difference between irregular chaotic patterns exhibited on the phase portraits of second-order double-loop SDMs and second-order interpolative bandpass SDMs. We have shown that by computing the difference between two consecutive state vectors, and plotting one component of the vectors against the other component, a simple regular pattern is resulted on the phase portrait for second-order double-loop SDMs. However, irregular chaotic patterns are still exhibited on the phase portrait for second-order interpolative bandpass SDMs. It is found that the Lyapunov exponent of state vectors of the second-order double-loop SDM is higher than that of the second-order interpolative bandpass SDM, while that of the transformed vectors of the second-order double-loop SDM is negative and 
much smaller than that of the second-order interpolative bandpass SDM. Also, the Lyapunov exponent of the transformed vectors increases for the second-order interpolative bandpass SDM. Hence, the proposed simple method can be used to distinguish these two types of SDMs from their state vectors without solving the state equations and the information of input signals.

\section{ACKNOWLEDGEMENT}

The work obtained in this paper was supported by a research grant from Queen Mary, University of London.

\section{REFERENCES}

[1] James C. Candy, “A use of limit cycle oscillations to obtain robust analog-todigital converters,” IEEE Transactions on Communications, vol. COM-22, no. 3, pp. 298-305, 1974.

[2] Orla Feely and Leon O. Chua, "The effect of integrator leak in $\Sigma-\Delta$ modulation,” IEEE Transactions on Circuits ans Systems, vol. 38, no. 11, pp. 1293-1305, 1991.

[3] Søren Hein, "Exploiting chaos to suppress spurious tones in general double-loop $\Sigma \Delta$ modulators," IEEE Transactions on Circuits ans Systems-II: Analog and Digital Signal Processing, vol. 40, no. 10, pp. 651-659, 1993.

[4] Ronan Farrell and Orla Feely, "Bounding the integrator outputs of second-order sigma-delta modulators," IEEE Transactions on Circuits ans Systems-II: Analog and Digital Signal Processing, vol. 45, no. 6, pp. 691-702, 1998.

[5] Orla Feely, “A tutorial introduction to non-linear dynamics and chaos and their application to sigma-delta modulators," International Journal of Circuit Theory and Applications, vol. 25, no. 5, pp. 347-367, 1997.

[6] Alan Wolf, Jack B. Swift, Harry L. Swinney and John A. Vastano, “Determining Lyapunov exponents from a time series,” Physica D, vol. 16, pp. 285-317, 1985.

[7] A. Lo Schiavo and A. M. Luciano, "Powerful and flexible fuzzy algorithm for nonlinear dynamic system identification,” IEEE Transactions on Fuzzy Systems, vol. 9, no. 6, pp. 828-835, 2001.

[8] X. M. Ren, A. B. Rad, P. T. Chan and Wai Lun Lo, "Identification and control of continuous-time nonlinear systems via dynamic neural networks,” IEEE Transactions on Industrial Electronics, vol. 50, no. 3, pp. 478-486, 2003. 